\title{
Sequential reactivity of serum against cyst antigens in Toxoplasma infection
}

\author{
G Woodison, A H Balfour, J E Smith
}

\begin{abstract}
Aims-To compare the recognition of Toxoplasma gondii tachyzoites and cysts by sera, from 10 patients.

Methods-Recognition of antigens from purified tachyzoites (RH strain) and bradyzoites (18691 strain) was compared using western immunoblotting. Sequential serum samples from 10 patients and one laboratory acquired RH infection were used.

Results-Recognition of cyst antigens was relatively low and occurred late in infection. The two stages were antigenically distinct with only a few shared bands.

Conclusion-Immunological recognition of the cystic stage of $T$ gondii is low. This implies that either cysts are poorly immunogenic or that cyst antigen is not available for processing and presentation.
\end{abstract}

(F Clin Pathol 1993;46:548-550)

Toxoplasma gondii is a common protozoan parasite affecting man and domestic animals. After infection there is a transient proliferative phase followed by a chronic phase in which infection is maintained by dormant tissue cysts (bradyzoites). In normal adults the disease is self-limiting, but in immunosuppressed subjects recrudescence of the proliferating forms may lead to serious or fatal toxoplasmosis. ${ }^{1}$ With the advent of AIDS, the incidence of cerebral toxoplasmosis has increased and has now become a major concern. Navia et al estimate that $30 \%$ of HIV infected patients with positive Toxoplasma serology will develop cerebral toxoplasmosis. ${ }^{2}$

Verhofstede, Van Gelder, and Rabaey followed the evolution of the IgG antibody response to tachyzoite antigens in sequential sera of acutely infected patients. ${ }^{3}$. The profile of antigen recognition changed during infection and they claimed that this could be used as a marker of acute or chronic disease. In AIDS associated Toxoplasma encephalitis, however, Weiss et al could find no consistent pattern of recognition. ${ }^{4}$

A major omission from these studies was the failure to examine immune recognition of bradyzoite antigens which are most relevant in chronic disease. Immune surveillance of cystic stages may be crucial to maintenance of these parasites and prevention of recrudes- cence. In the current study we examined the sequential development of antibodies to bradyzoite and tachyzyoite antigens.

\section{Methods}

The cystic strain of Toxoplasma gondii used in the study was a recent human isolate 18691 . The strain was maintained by repeated monthly passage in adult female outbred TUCKS No 1 mice, and infection was instigated through intraperitoneal injection of 200 cysts. For biochemical analysis, cysts were harvested from infected brain tissue. Mice were drained of blood and brains were pooled, homogenised by repeated passage through a 20 gauge needle, and purified on $45 \%$ Percoll gradients according to the method of Cornelissen, Overdulve, and Hoenderboom. ${ }^{5}$ Cysts recovered from the gradients were washed in phosphate buffered saline (PBS), counted, and stored at $-70^{\circ} \mathrm{C}$. Tachyzoites were obtained from the peritoneal fluid of adult female mice infected three days earlier with the $\mathrm{RH}$ strain of $T$ gondii. Parasites were harvested in RPMI 1640 medium (Gibco, Scotland), purified on a cellulose CF 11 (Whatman) column, counted and stored at $-70^{\circ} \mathrm{C}$.

Ten sets of sequential serum samples were obtained from infected patients. Clinical symptoms ranged from general malaise to lymphadenopathy and retinochoroiditis. Samples were taken throughout infection at time points of up to one year after infection, with dye test titres ranging from 8 to 16000 (equivalent to $2-4000 \mathrm{IU} / \mathrm{ml}^{6}$ ). Serum samples from a laboratory acquired $\mathrm{RH}$ strain infection were taken at intervals between days 4 and 314 after infection, with dye test titres ranging from 8 to 8192 .

Samples of 10000 cysts and $5 \times 10^{5}$ tachyzoites were freeze thawed three times, heated to $95^{\circ} \mathrm{C}$ for 4 minutes in non-reducing buffer, and electrophoresed on $12 \%$ discontinuous polyacrylamide gels. ${ }^{7}$ After separation by sodium doceyl sulphate-polyacrylamide gel electrophoresis (SDS-PAGE) proteins were electrophoretically transferred to nitrocellulose filters at $210 \mathrm{~mA}$ for 4 hours. $^{8}$ Filters were then washed in PBS containing $0.05 \%$ $\mathrm{v} / \mathrm{v}$ Tween for 30 minutes, dried, and cut into strips. All strips were incubated at $28^{\circ} \mathrm{C}$ for 45 minutes in antisera ( 1 in 25 dilution in PBS $0.05 \%$ Tween), then in alkaline phosphatase conjugated anti-mouse IgG (Sigma). Antigens were shown by developing strips using NBT/BCIP (Sigma) as a substrate. 
Figure 1 Western blot analysis of Toxoplasma tachyzoites ( $R H$ strain) and cysts (18691) strain probed with sequential sera from 10 infected patients (A-7). The first strip of each pair is tachyzoites and the second, cysts. The dye test titre at each time point is indicated.

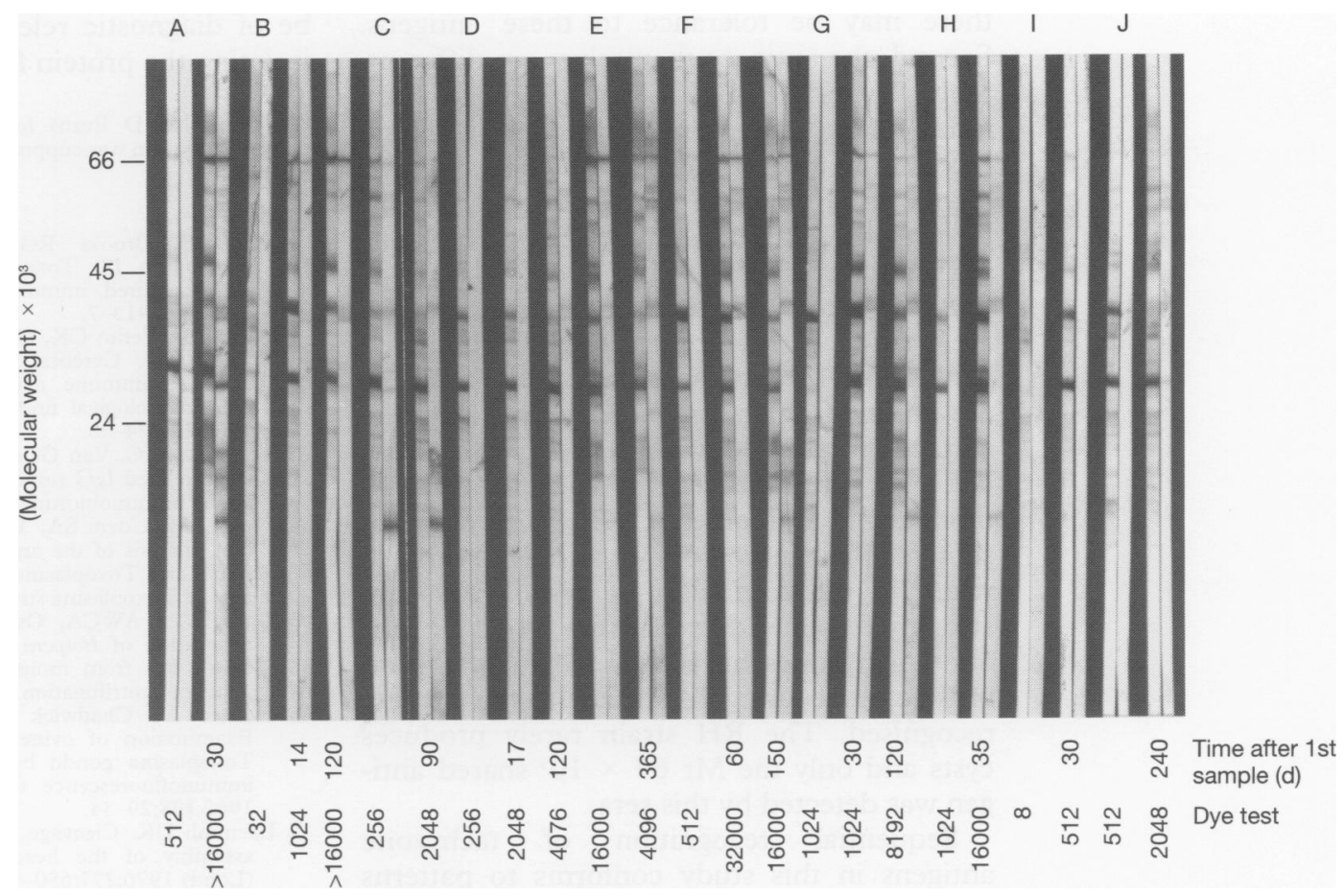

\section{Results}

Figure 1 shows the pattern of recognition of tachyzoite and cyst antigens with sequential sera from a panel of 10 infected subjects. Overall, there was considerable heterogeneity in antigen recognition by serum samples from different patients. With cysts, prominent antigens of Mr 69 and $17 \times 10^{3}$ were observed frequently during late stage infection. Other minor antigens were observed at $\mathrm{Mr}$ 63-58, $43,42,40,33,28.5,25$ and $23 \times 10^{3}$. Many of these antigens were apparent six weeks after infection. The first cyst antigen to appear was the shared tachyzoite/bradyzoite Mr $69 \times 10^{3}$ protein, P69, which could be detected as early as 20 days after infection. There was increased recognition of cyst

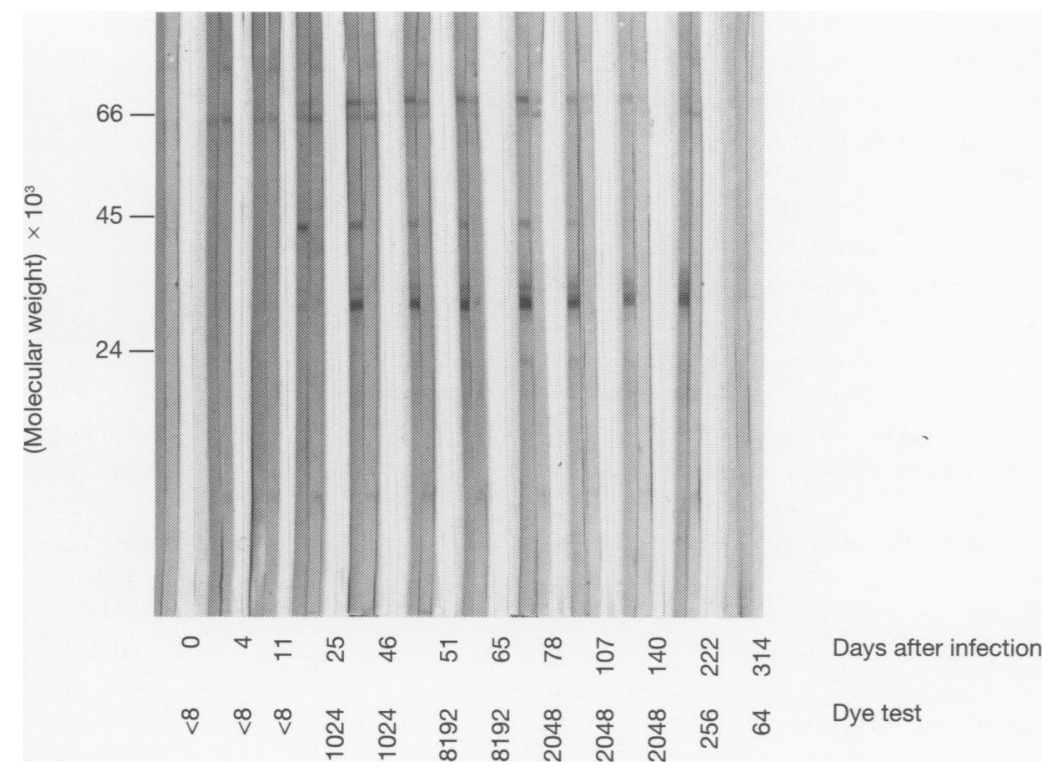

Figure 2 Western blot analysis of Toxoplasma tachyzoites (RH strain) and cysts (18691 strain) probed with sequential sera from a laboratory acquired $R H$ infection. Dye test titres are indicated. antigens with time as shown by an increase in both the intensity and number of bands.

By contrast, the intensity of antigen recognition in tachyzoites was very high, although recognition of some antigens decreased as infection progressed. A clear example of this was the reduced recognition of the dominant $\mathrm{Mr} 42 \times 10^{3}$ tachyzoite antigen (cases B, F, $\mathrm{G}$ and $\mathrm{J}$. The profile of tachyzoite antigens recognised is typical, with the earliest response being to the $\mathrm{Mr} \mathrm{42}$ and $30 \times 10^{3}$ antigens. $P 30$ could still be detected one year after infection.

Figure 2 shows the pattern of recognition of tachyzoites and cysts by sequential sera from an individual with a laboratory acquired infection ( $\mathrm{RH}$ strain) in which cysts are rarely formed. Overall, very few cyst antigens were recognised. In contrast, the response to tachyzoites was strong and the major surface antigens of $\mathrm{Mr} 42$ and $30 \times 10^{3}$ were, typically, the first to be recognised at 25 days after infection. With time, a complex of antigens were seen around P30, and at 70 days after infection, six antigens of $\mathrm{Mr} 33$, $32,31,30,29$ and $28 \times 10^{3}$ were observed. P30 could still be detected at 220 days after infection.

\section{Discussion}

The patient response to cyst antigens during natural infection varied greatly compared with the response against the proliferative tachyzoite. Two aspects should be emphasised. First, the antibody response to cysts was quantitatively lower than to tachyzoites. Several factors could have contributed to this poor response: cyst antigens may be inherently less immunogenic; antigen load may be lower; processing and presentation of cyst antigens, predominantly located in brain and muscle tissue, may be inefficient or indeed 
there may be tolerance to these antigens. Second, the antigens themselves are different. Stage specific antigens have already been noted using hyperimmune serum in crossrecognition experiments. ${ }^{9-11}$ The antigens recognised by human sera in this study conform to the $\left(\mathrm{Mr} \mathrm{67,28}\right.$ and $\left.26 \times 10^{3}\right)$ antigens described before. ${ }^{12}$

As shown by patient sera, some antigens are unique to bradyzoites, some are shared (P69 and P42), and some tachyzoite antigens are absent from cysts. The major tachyzoite surface protein P30 was not observed on cysts either by immune sera or specific anti-P30 monoclonal antibodies (C1E3, 1E5, data not shown). Recognition of cyst antigens seems to relate to the formation of cysts. Cysts are strongly recognised by late sera, implying that once tachyzoites have encysted in brain and muscle tissue, antigens specific to the cyst are recognised. The $\mathrm{RH}$ strain rarely produces cysts and only the $\mathrm{Mr} 69 \times 10^{3}$ shared antigen was detected by this sera.

Sequential recognition of tachyzoite antigens in this study conforms to patterns reported before. ${ }^{31314}$ The earlier IgG response is directed against molecules of $\mathrm{Mr} 42$ and 30 $\times 10^{3}$ which may represent major surface proteins. Many other antigens are recognised later, in particular around the $\mathrm{Mr} 60$ and $30 \times 10^{3}$ regions.

The $\mathrm{Mr} 69$ and $17 \times 10^{3}$ antigens seem to be the most relevant to the chronic stage and are recognised by most sera. The $\mathrm{Mr}$ $17 \times 10^{3}$ antigen was particularly prominent late in infection and is expressed only in cysts. This antigen may be useful in the diagnosis of chronic infection. The $\mathrm{Mr} 69 \times 10^{3}$ antigen is strongly immunogenic in both tachyzoites and cysts and shows no straindependent variation. ${ }^{12}$ This antigen may also be of diagnostic relevance. We are currently isolating the protein for sequence analysis.

We thank Ms D Evans for excellent technical assistance. Gillian Woodison was supported by an SERC studentship.

1 Luft BJ, Brooks RG, Conley FK, McCabe RE, Remington JS. Toxoplasmic encephalitis in patients with acquired immune deficiency syndrome. $¥ A M A$ 1984;252:913-7.

2 Navia BA, Petito CK, Gold JWM, Cho E-S, Jordan BD, Price RW. Cerebral toxoplasmosis complicating the acquired immune deficiency syndrome: clinical and acquired immune deficiency syndrome: clinical and neuropathologica

3 Verhofstede C, Van Gelder P, Rabaey M. The infectionstage-related IgG response to Toxoplasma gondii studied by immunoblotting. Parasitol Res 1988;74:516-20.

4 Weiss LM, Udem SA, Tanowitz H, Wittner M. Wester blot analysis of the antibody response of patients with AIDS and Toxoplasma encephalitis: Antigenic diversity among Toxoplasma strains. f Infect Dis 1988;157:7-13.

5 Cornelissen AWCA, Overdulve JP, Hoenderboom JM Separation of Isospora (Toxoplasma) gondii cysts and cystozoites from mouse brain by continuous densitygradient centrifugation. Parasitology 1981;83:103-8.

6 Hunter D, Chadwick $\mathrm{P}$, Balfour AH, Bridges JB. Examination of ovine foetal fluids for antibodies to Toxoplasma gondii by the dye test and an indirect immunofluorescence test specific for IgM. Br Vet $\mathcal{f}$ immunofluorescenc

7 Laemmli UK. Cleavage of structural proteins during the assembly of the head of bacteriophage $\mathrm{T}_{4}$. Nature (Lond) $1970 ; 277: 680-5$.

8 Towbin H, Stahelin T, Gordon J. Electrophoretic transfer of proteins from polyacrylamide gels to nitrocellulose heets: procedure and some applications. Proc Natl Acad Sci USA 1978;76:4350-4.

9 Lunde MN, Jacobs L. Antigenic differences between endozoites and cystozoites of Toxoplasma gondii. endozoites and cystozoite

10 Kasper LH, Ware PL. Recognition and characterisation of stage-specific oocyst/sporozoite antigens of Toxoplasma gondii by human antisera. $\mathcal{f}$ Clin Invest $1985 ; 75: 1570-7$.

$11 \mathrm{Kasper} \mathrm{LH}$. Identification of stage-specific antigens of Toxoplasma gondii. Infect Immun 1989;57:668-72.

12 Woodison G, Smith JE. Identification of the dominant cyst antigens of Toxoplasma gondii. Parasitology 1990 100:389-92.

13 Potassman I, Araujo FG, Desmonts GS, Remington JS Analysis of Toxoplasma gondii antigens recognised by human sera obtained before and after acute infection. $f$ Infect Dis 1986;154:650-7.

14 Decoster A, Darcy F, Capron A. Recognition of Decoster A, Darcy F, Capron A. Recognition of
Toxoplasma gondii excreted and secreted antigens by human sera from acquired and congenital toxoplasmosis: identification of markers of acute and chronic infection. Clin Exp Immunol 1988;73:376-82. 